\title{
Investigation of optimal digital image correlation patterns for deformation measurement
}

\author{
G.F. Bomarito ${ }^{1}$, T.J. Ruggles ${ }^{2}$, J.D. Hochhalter ${ }^{1}$, and A.H. Cannon ${ }^{3}$ \\ ${ }^{1}$ NASA, Durability, Damage Tolerance, and Reliability Branch, Hampton, VA \\ ${ }^{2}$ National Institute of Aerospace, Hampton, VA \\ ${ }^{3} 1900$ Engineering, LLC, Clemson, SC
}

August 30, 2016

\section{Extended Abstract}

Digital image correlation (DIC) relies on the visible surface features of a specimen to measure deformation. When the specimen itself has little to no visible features, a pattern is applied to the surface which deforms with the specimen and acts as artificial surface features. Since recent pattern application methods, e.g., micro-stamping [1] and lithography [2] allow for the application of highly customized patterns and because the accuracy and precision of DIC is dependent upon the applied pattern [3-6], an ideal pattern is sought for which the error introduced into DIC measurements is minimal. It is the goal of the present work to develop and refine an optimization technique to produce this type of DIC pattern.

The DIC literature has many examples of pattern quality metrics that aim to quantify the suitability of a pattern for DIC [e.g., 4-14]; however, most of these metrics have been developed with the intent of comparing pseudo-random patterns to each other. For that purpose, the quality metrics are well suited but typically do not generalize well to near-optimial patterns [15]. An example of this is the quality metric sum of square of subset intensity gradient [6-9], denoted SSSIG, which achieves a best value for a regular checkerboard pattern. Checkerboard patterns are ill-suited for DIC because they pose non-uniqueness problems. By using multiple pattern quality metrics simultaneously, Bomarito et al. [15] showed that these deficiencies can be avoided. They constructed an optimization method which centered around a quality metric $Q$ which is a combination of two metrics:

$$
Q=w_{1} \hat{S}+w_{2}\left(\hat{A}_{2}\right)^{n}
$$

where $\hat{S}$ is derived from SSSIG and $\hat{A}_{2}$ is the secondary auto-correlation peak height similar to the metric developed by Stoilov et al. [12]. In the minimization of $Q$, the $\hat{S}$ term is intended to be the primary contributor and the $\hat{A}_{2}$ term is meant to act as a constraint that ensures the pattern retains some local uniqueness (i.e., that it avoids regular checkerboard-type patterns). $w_{1}, w_{2}$ and $n$ are tuning variables that adjust the shape and relative intensity of the constraint.

In order to choose optimal values of $w_{1}, w_{2}$, and $n$, a study is performed which searches for the ideal balance of the two terms in Equation 1. In the study, several patterns are generated ranging from near-checkerboard to completely random which are then subjected to a numerical experiment. The experiment consists of the following steps:

1. The pattern is magnified to a desired resolution of 5 pixels per speckle $[16,17]$.

2. The contrast of the image is reduced, simulating imperfect lighting conditions: i.e., the grayscale values are changed from 0.0 and 1.0 to 0.25 and 0.75 .

3. Several numerical deformations are applied to the image. 
4. A Gaussian blur is applied to the patterns to simulate more realistic transitions from dark to light pixels.

5. Zero-mean Gaussian noise with standard deviation of $2 \%$ is added to the image to simulate random error in the imaging process.

6. DIC is performed on each image and compared to the applied deformations Step 3.

The result of the experiment is a distribution of DIC measurement errors for each of the input patterns.

For patterns that are closer to a checkerboard, small regions of spurious results are observed under some deformations. This is caused by the confusion of very similar subsets, and indicates that the results of the pattern may not be fully trusted when the true deformation is not known a priori. However, the general trend was that the more checkerboardlike patterns had higher accuracy and precision in areas where the spurious results did not present themselves. Using these results, an appropriate ratio could be identified between the two terms in Equation 1, which accompanied minimal error introduction while avoiding spurious results. The values of $w_{1}, w_{2}$, and $n$ are then calibrated.

Using the updated values of $w_{1}, w_{2}$, and $n$, the pattern optimization space is then explored using multiple initial points. Through this exploration, confidence is increased that a pattern more nearly the global minimum is found. Using the same numerical experiment outlined above, the optimized pattern is shown to have DIC measurement errors consistent with the best regions of the near-checkerboard patterns but without any incidents of spurious results. The pattern performs approximately $25 \%$ better than a random pattern.

Keywords: digital image correlation, pattern, optimization, quality metric, designer patterns

\section{References}

[1] A H Cannon, J D Hochhalter, A W Mello, G F Bomarito, and M D Sangid. Microstamping for improved speckle patterns to enable digital image correlation. Microscopy and Microanalysis, 21(S3):451-452, 2015.

[2] A D Kammers and S Daly. Small-scale patterning methods for digital image correlation under scanning electron microscopy. Measurement Science and Technology, 22(12):125501, 2011.

[3] H Haddadi and S Belhabib. Use of rigid-body motion for the investigation and estimation of the measurement errors related to digital image correlation technique. Optics and Lasers in Engineering, 46(2):185-196, 2008.

[4] D Lecompte, A Smits, S Bossuyt, H Sol, J Vantomme, D Van Hemelrijck, and AM Habraken. Quality assessment of speckle patterns for digital image correlation. Optics and Lasers in Engineering, 44(11):1132-1145, 2006.

[5] Y Sun and J H Pang. Study of optimal subset size in digital image correlation of speckle pattern images. Optics and Lasers in Engineering, 45(9):967-974, 2007.

[6] B Pan, H Xie, Z Wang, K Qian, and Z Wang. Study on subset size selection in digital image correlation for speckle patterns. Optics Express, 16(10):7037-7048, 2008.

[7] B Pan, Z Lu, and H Xie. Mean intensity gradient: an effective global parameter for quality assessment of the speckle patterns used in digital image correlation. Optics and Lasers in Engineering, 48(4):469-477, 2010.

[8] M A Sutton, J J Orteu, and H Schreier. Image correlation for shape, motion and deformation measurements: basic concepts, theory and applications. Springer Science \& Business Media, 2009.

[9] Y Q Wang, M A Sutton, H A Bruck, and H W Schreier. Quantitative error assessment in pattern matching: effects of intensity pattern noise, interpolation, strain and image contrast on motion measurements. Strain, 45 (2):160-178, 2009.

[10] T Hua, H Xie, S Wang, Z Hu, P Chen, and Q Zhang. Evaluation of the quality of a speckle pattern in the digital image correlation method by mean subset fluctuation. Optics 83 Laser Technology, 43(1):9-13, 2011.

[11] K Triconnet, K Derrien, F Hild, and D Baptiste. Parameter choice for optimized digital image correlation. Optics and Lasers in Engineering, 47(6):728-737, 2009. 
[12] G Stoilov, V Kavardzhikov, and D Pashkouleva. A comparative study of random patterns for digital image correlation. Journal of Theoretical and Applied Mechanics, 42(2):55-66, 2012.

[13] G Crammond, S W Boyd, and J M Dulieu-Barton. Speckle pattern quality assessment for digital image correlation. Optics and Lasers in Engineering, 51(12):1368-1378, 2013.

[14] S Bossuyt. Optimized patterns for digital image correlation. Imaging Methods for Novel Materials and Challenging Applications, 3:239-248, 2013.

[15] G F Bomarito, J D Hochhalter, T J Ruggles, and A H Cannon. Increasing accuracy and precision of digital image correlation through pattern optimization. Submitted to: Optics and Lasers in Engineering, 2016.

[16] B Winiarski, G S Schajer, and P J Withers. Surface decoration for improving the accuracy of displacement measurements by digital image correlation in SEM. Experimental Mechanics, 52(7):793-804, 2012.

[17] D Lecompte, S Bossuyt, S Cooreman, H Sol, and J Vantomme. Study and generation of optimal speckle patterns for dic. Proceedings of the Annual Conference and Exposition on Experimental and Applied Mechanics, 3:1643-1649, 2007. 